Physiology

\&

BEHAVIOR

\title{
Male-induced estrus synchronization in the female Siberian hamster (Phodopus sungorus sungorus)
}

\author{
James C. Dodge *, Mark B. Kristal, Lori L. Badura ${ }^{1}$ \\ Behavioral Neuroscience Program, Department of Psychology, University at Buffalo, Buffalo, NY, USA \\ Received 17 January 2002; received in revised form 22 April 2002; accepted 30 May 2002
}

\begin{abstract}
Olfactory cues play an integral role in the organization of events that mediate reproductive success. In a variety of species, priming pheromones, in particular, are important for ensuring reproductive fitness. To date, very little research has focused on how male-emitted priming pheromones, such as those that regulate the onset of puberty and estrus synchronization in females, affect the reproductive physiology of the female Siberian hamster (Phodopus sungorus sungorus). This lack of research may be due to the physiology of the Phodopus genus; vaginal cytology cannot be used as a reliable indicator of estrus or ovulation. Using a jugular cannulation technique to determine estrous stage by blood analysis of prolactin and luteinizing hormone, we sought to determine if male priming pheromones affect estrous cyclicity in the female Siberian hamster and, if so, whether the production of these priming pheromones is androgen dependent. Our results showed that females exposed to bedding from mature, intact males showed a significantly higher incidence of proestrus 3 days later than did females exposed to the bedding of mature, gonadectomized males. Therefore, we found that not only do male Siberian hamsters emit chemical signals that induce estrus synchronization, but also that this ability is likely to be androgen dependent.
\end{abstract}

(C) 2002 Elsevier Science Inc. All rights reserved.

Keywords: Pheromones; Estrus synchronization; Estrous cyclicity; Serial blood sampling; Prolactin; Luteinizing hormone; Siberian hamster; Whitten effect

\section{Introduction}

Reproductive fitness in mammals involves the successful integration of physiological, social, and environmental stimuli. Pheromones, particularly in nocturnal species or burrowing mammals such as the Siberian hamster (Phodopus sungorus sungorus), play a vital role in the coordination of events that ensure successful reproduction. Pheromones are historically categorized into two main divisions, signaling pheromones and priming pheromones $[2,26]$. Priming pheromones serve to modulate more long-term physiological or developmental changes that promote reproductive success, such as regulating the onset of puberty and estrus synchronization $[14,23]$. Priming pheromones emitted by males

* Corresponding author. Department of Biology, Center for Neuroendocrine Studies, Morrill Science Center, University of MassachusettsAmherst, Amherst, MA 01003, USA. Tel.: +1-413-577-3794; fax: +1-413545-3243.

E-mail address: jdodge@bio.umass.edu (J.C. Dodge).

${ }^{1}$ Current address: Department of Comparative and Experimental Medicine, Pfizer Global Research and Development, Grotton, CT, USA. appear to be androgen dependent, in that intact male mice are capable of advancing the onset of puberty in juvenile female mice, whereas castrated male mice are not [7].

Whitten's early work on mice was the first to demonstrate estrous cycle synchronization by a reproductively mature male. When group-housed female mice were introduced to a reproductively mature male, or placed in the presence of soiled bedding of a mature male, a synchronous wave of estrus appeared 3 days later [24,25]. Induction of ovulation cycle synchrony by the male has since been described in goats [3,21], sheep [12], prairie voles $[1,4,16,27]$, the short-tailed opossum [8], and humans $[10,17,19,22]$, but not in hamsters (i.e., using a physiologically based assessment). Male-induced estrus synchronization in hamsters would support the hypothesis that regulation of female reproductive physiology by male olfactory cues is a common feature of mammalian reproductive physiology.

Demonstration of male-induced estrous synchronization in either Djungarian (P. campbelli) or Siberian ( $P$. sungorus sungorus) hamsters appears to be an unlikely event because vaginal cytology in both of these species shows 
a mixed profile that renders discrimination of discrete stages of the estrous cycle impossible $[13,15,20,28]$. This is particularly troublesome for experiments that depend upon time breeding or controlling for stage of the estrous cycle of the female hamsters. Nevertheless, evidence drawn from behavioral measures indicates that male hamster olfactory cues may regulate reproductive physiology in females: Djungarian female hamsters display a higher incidence of mating on the third day after pairing with a male [5]. It remains to be established, however, whether male hamster olfactory cues regulate female hamster reproductive physiology directly. Recently, we devised an alternative physiological measure (i.e., one that does not rely on vaginal cytology) for characterizing estrous cycle stage in Siberian hamsters. Estrous state is determined after examining uterine weight and determining serum levels of prolactin and luteinizing hormone (LH) in serially drawn blood samples. There is a wellestablished relationship between uterine weight and plasma estrogen levels across the estrous cycle in both rats and hamsters $[9,28]$. Concurrent afternoon surges in prolactin and $\mathrm{LH}$, and uterine weight values intermediate between those of diestrus and estrus, are indicative of proestrus. In our protocol [6], hamster blood is sampled remotely via a surgically implanted jugular cannula to preclude stress-induced fluctuations in circulating prolactin levels.

In the present experiment, the use of this method of estrous stage characterization has allowed us to determine whether male hamster olfactory cues regulate female reproductive physiology (i.e., induction of estrus). By comparing the influence of intact males and castrated males on the estrous cycle, this experiment also determined whether the ability of the male hamster to induce estrus synchronization through olfactory cues is androgen dependent.

\section{Method}

\subsection{Animals and housing}

Adult male (intact, $n=4$; gonadectomized, $n=4$ ) and female $(n=12)$ Siberian hamsters, 65 days old and weighing approximately $35 \mathrm{~g}$, were obtained from our long-day $(16 \mathrm{~L} /$ $8 \mathrm{D}$, lights off at $1800 \mathrm{~h}$ ) breeding colony at the University at Buffalo. Hamsters were group-housed in $30 \times 30 \times 60$ $\mathrm{cm}$ plastic cages (male and female cages were positioned on opposite sides of the colony room), and were maintained in the long-day photoperiod at a temperature of $25-27^{\circ} \mathrm{C}$. The stability of the light/dark cycle and of colony temperatures was monitored daily. Hamster testing was performed outside of the colony room. All hamsters had access to food (Agway Lab Chow 3000) and water ad libitum. The University at Buffalo Institutional Animal Care and Use Committee approved all procedures prior to implementation.

\subsection{Olfactory cue manipulation and serial blood sampling procedure}

Test females were transferred (between 0900 and $1000 \mathrm{~h}$ ) either to a cage that contained soiled bedding from four reproductively mature, intact (INT) male hamsters ( $n=7$ test females) or from four gonadectomized (GNX) males $(n=5$ test females). Approximately $70 \mathrm{~h}$ later (between 0800 and $1000 \mathrm{~h}$ ), the female hamsters were anesthetized with a mixture of $25 \%$ halothane and $75 \%$ mineral oil, and surgically fitted with an indwelling jugular cannula as previously described [6]. The females were permitted to recover from surgery from 1000 to $1300 \mathrm{~h}$. Starting at 1300 $\mathrm{h}$, the females were subjected to the blood sampling procedure $(80 \mu \mathrm{l})$ each hour, and serum was extracted for analysis of circulating levels of prolactin and $\mathrm{LH}$ using radioimmunoassay (RIA). Shortly after the last blood sample was taken $(1700 \mathrm{~h})$, each female hamster was euthanized by anesthetic overdose and had her uterus removed and weighed. Males had undergone gonadectomy under anesthesia (ketamine $100 \mathrm{mg} / \mathrm{kg}$; xylazine $20 \mathrm{mg} / \mathrm{kg}$ ) approximately 4 weeks before females were exposed to their bedding.

\subsection{Determination of estrous cyclicity}

Estrous cyclicity is difficult to monitor in this species. Unlike many other rodent species, Siberian hamsters show no clear-cut changes in the profile of vaginal cell types, or in the characteristics of vaginal discharge. Therefore, the estrous cycle of hamsters in this study was determined by assessing both the uterine weight at the time of euthanasia and the presence or absence of an LH and prolactin surge during the afternoon of sampling. By using this combination of indices, it was possible to assign the females to diestrus, estrus, and proestrus groups using the following criteria: estrus $=$ no LH or prolactin surge and large, engorged uteri; diestrus $=$ no LH or prolactin surge and small uteri; proestrus $=$ an afternoon surge in $\mathrm{LH}$ and prolactin and uteri of moderate size.

\subsection{RIA}

RIAs were completed using a previously established protocol [6]. For prolactin analysis, serum $(20 \mu \mathrm{l})$ from each blood sample was dispensed with $280 \mu \mathrm{l}$ of $0.4 \%$ bovine serum albumin- $0.1 \%$ gelatinized phosphate-buffered saline $(\mathrm{pH} 7.0)$ into borosilicate tubes for RIA. Guinea pig antihamster prolactin $(1: 100,000)$, obtained from Dr. A.F. Parlow, served as the first antibody, and goat-antiguinea pig gamma globulin (1:15; Chemicon) was used as the precipitating secondary antibody. Purified hamster prolactin (obtained from Dr. A.F. Parlow) was iodinated using the chloramine $\mathrm{T}$ method and served as trace. Serial dilutions of this hormone were used as the standard reference preparation. Pooled sera from lactating females, long-day 
exposed females, and long-day exposed males were run throughout the assay to calculate intraassay variability. The lower limit of detectability of the assay was $0.5 \mathrm{ng} / \mathrm{ml}$ and the coefficients of variance at $40 \%, 60 \%$, and $70 \%$ bound were $5.5 \%, 4.2 \%$, and $5 \%$, respectively.

For LH analysis, the same procedures were used, except that the samples were dispensed with $0.1 \%$ gelatinized phosphate-buffered saline ( $\mathrm{pH}$ 7.0). Primary antibody, obtained from the NIDDK, consisted of rabbit-antirat LH (NIDDK Anti-rLH-S-11; 1:100,000). The secondary antibody was goat-antirabbit gamma globulin (1:15; Chemicon). Purified rat $\mathrm{LH}$ was iodinated using the chloramine $\mathrm{T}$ method to serve as trace, and serial dilutions of this hormone served as the standard reference preparation. The lower limit of detectability for the assay was $0.1 \mathrm{ng} / \mathrm{ml}$, and the intraassay coefficients of variance at $50 \%, 60 \%$, and $80 \%$ bound were $3.7 \%, 2.5 \%$, and $5.2 \%$, respectively.

\subsection{Statistics}

Prolactin and LH levels were analyzed with two-way mixed model ANOVAs (Hormone $\times$ Sampling time) with repeated measures on the sampling time variable. Significant interactions were broken down with analysis of simple main effects. Main effects were further analyzed by Fisher's LSD post-hoc tests where appropriate. Uterine weights were analyzed with a one-way ANOVA (long-day diestrus, longday proestrus) followed by Fisher's LSD post-hoc tests. All comparisons were considered significant if $P<.05$. The number of female hamsters in the two exposure groups classified as being in proestrus was analyzed using an independent Chi-square test because $25 \%$ of the females, at most, would be expected to be in proestrus based on a

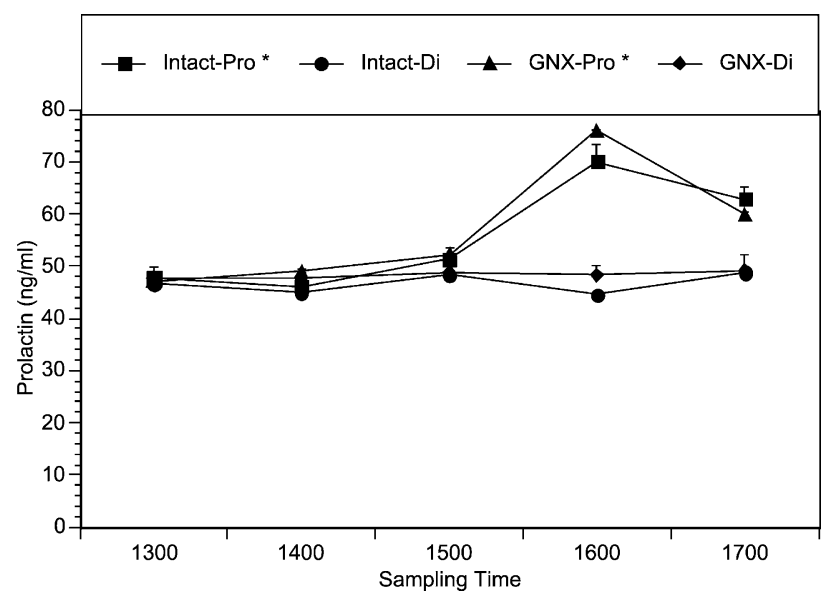

Fig. 1. Prolactin values (mean \pm S.E.M., $\mathrm{ng} / \mathrm{ml}$ ) for female hamsters classified as being in diestrus after exposure to soiled bedding from either intact males (Intact-Di) or from gonadectomized males (GNX-Di), and for females classified as being in proestrus after exposure to soiled bedding from either intact males (Intact-Pro) or gonadectomized males (GNX-Pro). * Significant elevation in prolactin for both proestrus groups at the 1600-h sampling point vs. diestrus groups $[F(1,8)=28.12, P<.0001]$.

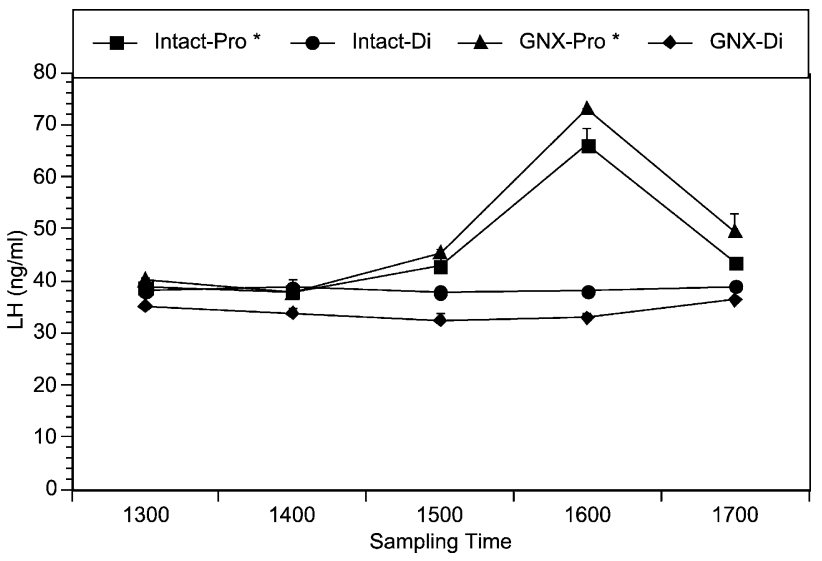

Fig. 2. LH values (mean \pm S.E.M., $\mathrm{ng} / \mathrm{ml}$ ) for female hamsters classified as being in diestrus after exposure to soiled bedding from either intact males (Intact-Di) or from gonadectomized males (GNX-Di), and for females classified as being in proestrus after exposure to soiled bedding from either intact males (Intact-Pro) or gonadectomized males (GNX-Pro). * Significant elevation in LH for both proestrus groups at the 1600-h sampling point vs. diestrus groups $[F(1,8)=58.25, P<.0001]$.

random distribution of rats among four equal length stages of the cycle.

\section{Results}

\subsection{LH levels}

Female hamsters classified as being in diestrus did not show fluctuations in LH across the sampling period in either exposure group (one of seven exposed to the bedding of intact males was found to be in diestrus; three of five exposed to the bedding of gonadectomized males were found to be in diestrus). In contrast, those female hamsters classified as being in proestrus (six of seven exposed to the bedding of intact males; two of five exposed to the bedding

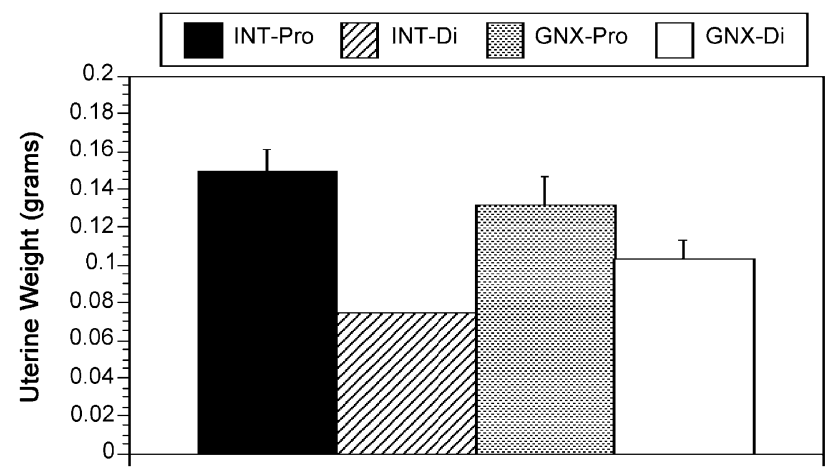

Fig. 3. Uterine weights (mean \pm S.E.M., $\mathrm{mg}$ ) for female hamsters classified as being in diestrus after exposure to soiled bedding from either intact males (Intact-Di) or from gonadectomized males (GNX-Di), and for females classified as being in proestrus after exposure to soiled bedding from either intact males (Intact-Pro) or gonadectomized males (GNX-Pro). Significantly greater uterine weights for both proestrus groups as compared to the diestrus groups $(P<.01)$ for both comparisons. 


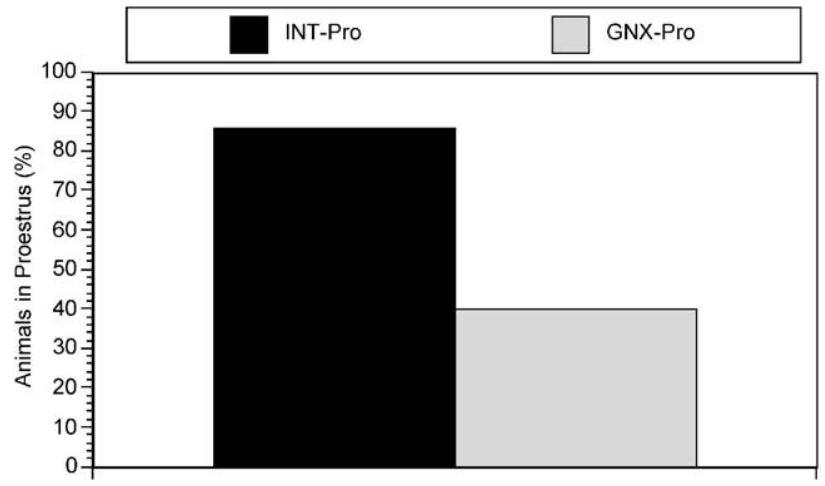

Fig. 4. Percentage of female hamsters in proestrus for both exposure groups. Significantly higher percentage of females in proestrus after exposure to soiled bedding from intact males, than after exposure to bedding of gonadectomized males $(P<.01)$.

of gonadectomized males) showed a significant elevation at the 1600-h sampling point when compared to their diestrus counterparts (see Fig. 1).

\subsection{Prolactin levels}

Prolactin levels across groups were similar to those obtained for LH. Female hamsters classified as being in diestrus showed constant prolactin levels across the sampling period, whereas those classified as being in proestrus showed a significant elevation at the 1600-h point (see Fig. 2).

\subsection{Uterine weights}

The uterine weight data revealed a significant effect of cycle stage, with females in proestrus having higher uterine weights than did females in diestrus from both exposure groups (see Fig. 3).

\subsection{Percentage of group in proestrus}

The Chi-square analysis revealed a significant difference from expected value $(25 \%)$ of females in proestrus in the group exposed to bedding of intact males $(85.7 \%, n=7)$, but not among females exposed to bedding of gonadectomized males $(40 \%, n=5)$ (see Fig. 4).

\section{Discussion}

Our results indicate that male hamster pheromonal cues regulate female reproductive physiology and that the ability to emit these cues is androgen dependent. Evaluation of circulating hormone levels and uterine weights of females exposed to the soiled bedding of either reproductively intact or gonadectomized mature males showed that exposure to the bedding of intact males was followed by a significantly greater proportion of females being in proestrus 3 days later. It should be noted that two of five females exposed to gonadectomized male bedding showed hormonal profiles and uterine weight that were characteristic of proestrus, but this incidence reflects the percentage of animals expected to be in proestrus by chance using random sampling procedures. Our current results are the first demonstration, using physiological measures, to demonstrate that male-induced estrus synchronization occurs in female Siberian hamsters (P. sungorus sungorus). This finding increases the generality of male-induced estrus synchronization among mammalian species and supports the possibility that this may be a common characteristic of mammalian reproductive physiology.

In recent years, a growing number of experimental observations have established that environmental stimuli play an important role in the control of several aspects of female hamster reproductive physiology. For example, female-induced estrus synchronization occurs within the Syrian hamster, an effect that is mediated by the dominant female of the social group [11]. In addition, exposure of recently weaned female Djungarian hamsters to an adult male accelerates the onset of puberty in those females by approximately 15 days [18]. Taken together with our current results, these reports verify that olfactory cues are functionally important in regulating female reproductive physiology - the timing of estrus, ovulation, and the onset of puberty can all be influenced by the odors of conspecifics. Olfactory cue-mediated regulation of female reproductive physiology may be particularly important in Siberian hamsters because they are seasonal breeders. Olfactory cues may have an important role in regulating both the onset and offset of the breeding season. The positive results obtained from this experiment have laid the groundwork for future experiments to test this hypothesis.

From a practical standpoint, our results show that estrus synchronization in Siberian hamsters, and therefore timed breeding, can be effected by preexposing the breeder female to mature, intact males or their bedding for 3 days prior to the introduction of the stud male. Therefore, experimenters, especially those interested in female reproductive behavior, the neurochemical regulation of gonadotropin release, or hormonal influences on neurochemical systems, can avoid the confound of having to prime female hamsters with exogenous hormones that may not be physiologically relevant.

In conclusion, the results of this experiment indicate that male-induced estrus synchronization occurs in the Siberian hamster, and that the male's ability to produce this synchronization appears to be androgen dependent.

\section{Acknowledgements}

Special thanks to the NIDDK and to Dr. A.F. Parlow for the generous donation of the reagents used in the RIAs. This work was supported, in part, by NSF grant IBN9603780 to 
Lori L. Badura, and internal grant funds from the Dean of the Faculty of Social Sciences at the University at Buffalo.

\section{References}

[1] Baddaloo EGY, Chulow FV. Effects of the male on growth, sexual maturation, and ovulation of young female meadow voles, Microtus pennsylvanicus. Can J Zool 1981;59:415-21.

[2] Bronson FH. Pheromonal influences on mammalian reproduction. In: Diamond M, editor. Perspectives in reproduction and sexual behavior. Bloomington: Indiana Univ. Press, 1968. p. 341-61.

[3] Coblentz B. Function of scent-urination in ungulates with special reference to feral goats (Capra hircus L.). Am Nat 1976;110:549-57.

[4] Dluzen DE, Ramierez VD, Carter CS, Getz LL. Male vole urine changes luteinizing hormone-hormone and norepinephrine in female olfactory bulb. Science 1981;212:573-5.

[5] Erb GE, Edwards HE, Jenkins KL, Mucklow LC, Wynne-Edwards KE. Induced components in the spontaneous ovulatory cycle of the Djungarian hamster (Phodopus campbelli). Physiol Behav 1993;54: 995-9.

[6] Dodge JC, Badura LL. Norepinephrine dialysate levels in the hypothalamic paraventricular nucleus: influence on photoperiod-driven prolactin levels in the female Siberian hamster. Neuroendocrinology 2001;73:102-10.

[7] Drickamer LC, Murphy RX. Female mouse maturation: effects of excreted bladder urine from juvenile and adult males. Dev Psychobiol 1978; $11: 63-72$.

[8] Fadem BH. Evidence for the activation of female reproduction by males in a marsupial, the gray tailed opossum (Monodephis domestica). Biol Reprod 1985;33:112-6.

[9] Feder HH. Estrous cyclicity in mammals. In: Adler NT, editor. Neuroendocrinology of reproduction: physiology and behavior. New York: Plenum, 1981. p. 279-348.

[10] Graham CA, McGrew WC. Menstrual synchrony in female undergraduates living in a coeducational campus. Psychoneuroendocrinology 1980;5:245-52.

[11] Handelmann G, Ravizza R, Ray WJ. Social dominance determines estrous entrainment among female hamsters. Horm Behav 1980;14: $107-15$.

[12] Knight TW, Tervit HR, Lynch PR. Effects of boar pheromones, ram's wool and the presence of bucks on ovarian activity in anovular ewes early in the breeding season. Anim Reprod Sci 1984;6:129-34.
[13] Lai SC, Vasilieva NY, Johnston RE. Odors providing sexual information in Djungarian hamsters: evidence for an across odor code. Horm Behav 1996;30:26-36.

[14] McClintock MK. Estrous synchrony and its mediation by airborne chemical communication (Rattus norvegicus). Horm Behav 1978;10: $264-76$.

[15] McMillan HJ, Wynne-Edwards KE. Divergent reproductive endocrinology of the estrous cycle and pregnancy in dwarf hamsters (Phodopus). Comp Biochem Physiol 1999;124:53-67.

[16] Milligan SR. Social environment and ovulation in the vole, Microtus agrestis. J Reprod Fertil 1974;41:35-47.

[17] Quadagno DM, Shubeita HE, Deck J, Francoer D. The effects of males, athletics activities and all female living conditions on the menstrual cycle. Psychoneuroendocrinology 1981;6:239-44.

[18] Reasner DS, Johnston RE. Acceleration of reproductive development in female Djungarian hamsters by adult males. Physiol Behav 1988; 43:57-64.

[19] Russel MJ, Switz GM, Thompson K. Olfactory influences on the human menstrual cycle. Pharmacol Biochem Behav 1980;13:737-8.

[20] Schlatt S, Niklowitz P, Hoffmann K, Nieschlag E. Influence of short photoperiods on reproductive organs and estrous cycles of normal and pinealectomized female Djungarian hamsters, Phodopus sungorus. Biol Reprod 1993;49:243-50.

[21] Shelton M. Goats: influence of various exteroceptive factors in initiation of estrus and ovulation. Int Goat Sheep Res 1980;1:156-62.

[22] Stern K, McClintock MK. Regulation of ovulation by human pheromones. Nature 1998;392:177-9.

[23] Vandenbergh JG. Pheromones and mammalian reproduction. In: Knobil E, Neill JD, editors. The physiology of reproduction. 2nd ed. New York: Raven, 1994. p. 343-59.

[24] Whitten WK. Modification of the oestrous cycle of the mouse by external stimuli associated with the male. J Endocrinol 1956;13: 399-404.

[25] Whitten WK. Occurrence of anoestrus in mice caged in groups. J Endocrinol 1959;18:102-7.

[26] Wilson ED, Bossert WA. Chemical communication among animals. Recent Prog Horm Res 1963;19:673-710.

[27] Witt DM, Carter CS, Carlstead K, Read LD. Sexual and social interactions preceding and during male induced oestrus in prairie voles, Microtus ochrogaster. Anim Behav 1988;36:1465-71.

[28] Wynne-Edwards KE, Terranova PF, Lisk RD. Cyclic Djungarian hamsters, Phodopus campbelli, lack the progesterone surge normally associated with ovulation and behavioral receptivity. Endocrinology 1987; 120:1308-16. 